\title{
Bioaérosols provenant de la respiration buccale : mode de transmission méconnu de la COVID-19?
}

\author{
Saravanakarthikeyan Balasubramanian ${ }^{1 \star}$, Divya Vinayachandran²
}

\section{Résumé}

Le monde entier a été touché par la pandémie de maladie à coronavirus 2019 (COVID-19), et de nombreux chercheurs se lancent dans une course pour comprendre l'évolution de la maladie et entreprendre des analyses de risque afin de formuler des stratégies de traitement efficaces. Le coronavirus du syndrome respiratoire aigu sévère 2 (SRAS-CoV-2) est très transmissible par la toux et les éternuements, ainsi que par la respiration et en parlant, ce qui peut expliquer la transmission virale à partir de porteurs asymptomatiques. Les bioaérosols produits pendant la respiration buccale, un processus expiratoire chez les personnes qui respirent habituellement par la bouche, doivent être considérés, en plus des bioparticules nasales, comme un mode de transmission potentiel de la COVID-19. Les professionnels de la santé buccodentaire craignent, à juste titre, le risque d'exposition dû à la proximité du contact direct et au mode de transmission. L'objectif de ce commentaire est de résumer les recherches menées dans ce domaine et de proposer des stratégies pour limiter la propagation de la COVID-19, notamment dans les cabinets dentaires.

Citation proposée : Balasubramanian S, Vinayachandran D. Bioaérosols provenant de la respiration buccale : Mode de transmission méconnu de la COVID-19? Relevé des maladies transmissibles au Canada 2021;47(5/6):302-5. https://doi.org/10.14745/ccdr.v47i56a05f

Mots-clés : bioaérosols, coronavirus, COVID-19, respiration buccale, diagnostic oral, risque de transmission
Cette oeuvre est mise à la disposition selon les termes de la licence internationale Creative Commons Attribution 4.0

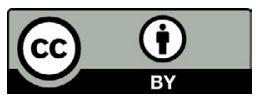

Affiliations

1 Département de dentisterie conservatrice et d'endodontie, SRM Dental College, Ramapuram, SRM Institute of Science and Technology, Chennai, Inde

2 Département de médecine orale et de radiologie, SRM Kattankulathur Dental College \& Hospitals, SRM Institute of Science and Technology, Chennai, Inde

\section{*Correspondance :}

skmdc2006@gmail.com

Note de l'éditeur : Au Canada, la prévalence de l'infection due à la COVID-19 dans la profession dentaire est très faible, probablement en raison de la grande quantité d'EPI disponible, de mesures de dépistage du patient et du nombre de formations obligatoires sur les techniques de prévention des infections. De plus, il est important de clarifier les concepts présentés dans cet article. Les particules expiratoires s'appliquent à la fois aux gouttelettes et aux bioaérosols. La différence entre les gouttelettes et les bioaérosols réside dans leur taille, qui a un impact sur leur distance potentielle de «déplacement» entre une personne infectée et la personne à risque qui passe ou qui se trouve à proximité. Voir: COVID-19 : Guide de ventilation des espaces intérieurs pendant la pandémie

\section{Introduction}

Le coronavirus du syndrome respiratoire aigu sévère 2 (SRAS-CoV-2), virus responsable de la maladie à coronavirus de 2019 (COVID-19), serait très transmissible par les gouttelettes respiratoires et aérosols émis lors de la toux, des éternuements, de la parole et du chant. Cet article se penche sur les procédures générant des aérosols dans les domaines médical et dentaire (1). Des rapports récents ont souligné le rôle possible de la transmission du SRAS-CoV-2 par les particules expiratoires émises lors d'activités normales de respiration et de parole $(2,3)$. Les aérosols biologiques ( $d$ 'une taille inférieure à $1 \mu \mathrm{m}$ ) sont également produits en grande quantité lors de la respiration buccale et l'impact de cette dernière doit également être pris en compte dans la transmission du SRAS-CoV-2 (4). Ceci est très pertinent dans un cabinet dentaire où les patients doivent ouvrir la bouche pendant un temps considérable, ce qui pose un risque de transmission de maladies par les bioparticules expirées. II n'y a actuellement aucun rapport de recherche ou examen sur cette voie de transmission. Notre commentaire présente donc un aperçu sur la respiration buccale et de son importance potentielle dans la transmission du SRAS-CoV-2, en particulier dans un cabinet dentaire. Cette question concerne tous les professionnels du secteur dentaire, y compris les dentistes, les hygiénistes dentaires et les assistants dentaires. En outre, nous soulignons quelques questions de recherche relatives aux particules expiratoires de la respiration buccale qui nécessitent des réponses urgentes par le biais de recherches appropriées afin de contrôler efficacement la transmission du SRAS-CoV-2 dans un contexte clinique. 


\section{Bioparticules exhalées et voies d'infection potentielles dans un cabinet dentaire}

Normalement, les voies potentielles d'infection de maladies transmissibles dans un cabinet dentaire comprennent le contact direct avec les fluides corporels (salive ou sang) d'un patient infecté, le contact avec les instruments ou les surfaces de l'environnement contaminés par les patients et par des particules d'aérosol infectieuses (5). Dans le cas de la COVID-19, les bioparticules exhalées peuvent être émises par les patients par le biais de diverses actions respiratoires, notamment la respiration par la bouche, la respiration par le nez, la toux et la parole. Pappineni et Rosenthal (6) ont étudié les gouttelettes exhalées par ces différents modes en utilisant un compteur optique de particules et un microscope électronique à transmission analytique. Les chercheurs ont rapporté que la quantité de gouttelettes émises était plus élevé en cas de toux, suivi par la respiration buccale, la respiration nasale et la parole, respectivement. Le nombre plus faible de bioparticules émises par la respiration nasale par rapport à la respiration buccale peut être attribué aux processus de filtration qui se produisent dans la cavité nasale (6). Ces gouttelettes de bioaérosols expiratoires sont polydispersées et la taille des gouttelettes a un impact énorme sur le processus de transmission des maladies $(2,4,6)$. Les plus petites gouttelettes s'évaporent rapidement et deviennent des «noyaux de gouttelettes " qui peuvent rester en suspension dans l'air pendant de longues périodes (7). Ces particules d'aérosol plus petites (de 0,5 à $10 \mu \mathrm{m}$ de diamètre) peuvent pénétrer et se loger dans les minuscules passages pulmonaires et provoquer un risque élevé d'infection (8).

\section{Respiration buccale : Un facteur de risque négligé de la COVID-19?}

De manière alarmante, la COVID-19 est également hautement transmissible chez les porteurs présymptomatiques ou non-symptomatiques $(9,10)$. La respiration buccale, qui se caractérise par l'inspiration et l'expiration par la bouche, résulte principalement d'une obstruction des voies aériennes supérieures ou lorsqu'elle devient un comportement chronique, elle est considérée comme une fonction respiratoire anormale. Les personnes qui respirent par la bouche sont celles qui ont des lèvres entrouvertes, sèches et fissurées, une langue antérieure, des muscles élévateurs mandibulaires faibles, un palais profond et étroit, des altérations dentaires et une croissance du visage essentiellement verticale (11).

Les causes les plus fréquemment rapportées de la respiration buccale sont la rhinite allergique chronique $(81,4 \%)$,

I'hypertrophie des adénoïdes (79,2\%), l'hypertrophie des amygdales $(12,6 \%)$ et la déviation de la cloison nasale $(1,0 \%)(12,13)$. Les principales manifestations cliniques des personnes qui respirent par la bouche sont les suivantes : sommeil à bouche ouverte (86\%), ronflement $(79 \%)$, démangeaisons nasales $(77 \%)$, bave de salive $(62 \%)$, problèmes de sommeil nocturne $(62 \%)$, obstruction nasale $(49 \%)$ et irritabilité générale (43\%) (13). En outre, de nombreux problèmes dentaires, notamment les caries dentaires, les maladies parodontales, I'halitose, les anomalies craniofaciales et la malocclusion, sont couramment observés chez les personnes qui respirent par la bouche (12-14).

Récemment, il a été signalé que l'oxyde nitrique nasal peut contribuer à réduire la charge virale du SRAS-CoV-2 et le risque de pneumonie causée par la COVID-19 en favorisant des mécanismes de défense antivirale plus efficaces dans les voies respiratoires (15). Cependant, la respiration buccale réduit considérablement l'efficacité de l'oxyde nitrique, ce qui diminue la réponse antivirale (contrairement à la respiration nasale). Cette observation souligne l'importance des bioparticules expirées par la bouche dans la transmission de la COVID-19, et l'importance de faire la distinction entre la bouche et le nez comme voies de transmission des bioaérosols (15).

Les dentistes et les autres professionnels de la santé buccodentaire travaillant dans un cabinet dentaire courent un risque élevé d'exposition à ces personnes asymptomatiques, qu'il s'agisse de personnes qui respirent par la bouche de manière chronique ou de celles qui doivent garder la bouche ouverte pendant un temps considérable lors d'un examen dentaire de routine. Comme il est bien connu que ces particules émises peuvent faciliter la propagation de diverses maladies infectieuses, y compris la grippe (16), il est à craindre qu'elles puissent également faciliter la propagation de la COVID-19. Étant donné que le profil de transmission du SRAS-CoV-2 pourrait ressembler davantage à celui de la grippe qu'à celui du syndrome respiratoire aigu sévère (SRAS), il convient de prendre en compte la menace que représentent les aérosols produits par la respiration buccale (en plus de la respiration nasale) (9). Le SRAS-CoV-2 et la grippe se caractérisent tous deux par une infectiosité accrue peu de temps après ou même avant l'apparition des symptômes, contrairement au SRAS, où I'infectiosité atteint son maximum environ 7 à 10 jours après l'apparition des symptômes. La transmission du SRAS-CoV-2 par les bioparticules expiratoires des individus présymptomatiques joue donc un rôle important (9). Cependant, un examen minutieux de la littérature a révélé qu'aucune étude n'avait considéré les bioaérosols produits lors de la respiration buccale comme un mode de transmission potentiel du SRAS-CoV-2, malgré sa grande plausibilité. Ce commentaire vise donc à encourager la recherche sur 1) le potentiel de transmission du SRAS-CoV-2 par les bioaérosols et 2) les méthodes permettant de limiter la transmission, notamment dans un cabinet dentaire. 


\section{Questions urgentes qui nécessitent une attention immédiate}

Les spécialistes des aérosols doivent répondre à de nombreuses questions importantes concernant la transmission du SRASCoV-2 par la respiration buccale (2). Les personnes infectées mais asymptomatiques qui respirent par la bouche émettent-elles plus de bioaérosols que les personnes en bonne santé qui respirent par la bouche? Les bioaérosols provenant de la respiration par la bouche sont-ils plus susceptibles de transmettre le SRAS-CoV-2 que les bioaérosols expirés normalement? Le prélèvement rapide d'échantillon de salive pour le SRAS-CoV-2 est-il un outil utile pour évaluer l'infectiosité des bioaérosols émis par des personnes en bonne santé, présymptomatiques ou asymptomatiques qui respirent par la bouche? Quelles sont les méthodes expérimentales optimales pour évaluer la virulence des bioaérosols? L'encadré 1 présente un résumé de ces questions.

\section{Encadré 1 : Points saillants}

- $\quad$ Le SRAS-CoV-2 se propage par les particules expiratoires émises lors de la respiration normale respiration normale, de la conversation, du chant, des cris, de la toux et des éternuements

- Les bioaérosols sont produits en plus grande quantité lors de la respiration par la bouche par rapport à la respiration normale

- Les aérosols provenant de la respiration buccale constituent un facteur de risque négligé et sous-estimé dans la transmission de la COVID-19

- En outre, il est nécessaire de disposer de plus de données sur la propagation de la COVID-19 au moyen de bioparticules expiratoires afin d'informer les mesures préventives et de contribuer à la lutte contre la pandémie

\section{Conclusion}

En conclusion, les bioaérosols provenant de la respiration buccale, en plus des particules expiratoires nasales, doivent également être considérés comme une source potentielle de transmission de la COVID-19. Cependant, des preuves concrètes de recherche clinique mettant en évidence le rôle des bioparticules respirées par la bouche dans la transmission de la COVID-19 sont obligatoires pour soutenir notre hypothèse, même si elle peut sembler logiquement vraie. De plus, avec I'augmentation considérable du nombre de cas de COVID-19 chaque jour, il est nécessaire de sensibiliser davantage les professionnels de la santé buccodentaire au processus de transmission de la maladie et aux facteurs de risque associés aux activités expiratoires normales, comme le mentionnent Anderson et al. (3).

\section{Déclaration des auteurs}

S. K. B. - Conceptualisation, rédaction-préparation du projet original

D. V. - Conceptualisation, rédaction-révision et édition

Le contenu de l'article et les points de vue qui y sont exprimés n'engagent que les auteurs et ne correspondent pas nécessairement à ceux du gouvernement du Canada.

\section{Intérêts concurrents}

Aucun.

\section{Financement}

Aucun.

\section{Références}

1. Organisation mondiale de la Santé. Questions-réponses : Comment se transmet la COVID-19? Genève, Suisse : OMS; 2020 (accédé 2021-03-23). https://www.who.int/ emergencies/diseases/novel-coronavirus-2019/question-andanswers-hub/q-a-detail/coronavirus-disease-covid-19-how-isit-transmitted

2. Asadi S, Bouvier N, Wexler AS, Ristenpart WD. The coronavirus pandemic and aerosols: does COVID-19 transmit via expiratory particles? Aerosol Sci Technol 2020;54(6):635-8. DOl PubMed

3. Anderson EL, Turnham P, Griffin JR, Clarke CC. Consideration of the Aerosol Transmission for COVID-19 and Public Health. Risk Anal 2020;40(5):902-7. DOI PubMed

4. Edwards DA, Man JC, Brand P, Katstra JP, Sommerer K, Stone HA, Nardell E, Scheuch G. Inhaling to mitigate exhaled bioaerosols. Proc Natl Acad Sci USA 2004;101(50):17383-8. DOI PubMed

5. Garner JS; The Hospital Infection Control Practices Advisory Committee. Guideline for isolation precautions in hospitals. Infect Control Hosp Epidemiol 1996;17(1):53-80. DOI PubMed

6. Papineni RS, Rosenthal FS. The size distribution of droplets in the exhaled breath of healthy human subjects. J Aerosol Med 1997;10(2):105-16. DOI PubMed

7. Chao CY, Wan MP, Morawska L, Johnson GR, Ristovski ZD, Hargreaves M, Mengersen K, Corbett S, Li Y, Xie X, Katoshevski D. Characterization of expiration air jets and droplet size distributions immediately at the mouth opening. J Aerosol Sci 2009;40(2):122-33. DOI PubMed 
8. Micik RE, Miller RL, Mazzarella MA, Ryge G. Studies on dental aerobiology. I. Bacterial aerosols generated during dental procedures. J Dent Res 1969;48(1):49-56.

DOI PubMed

9. He X, Lau EH, Wu P, Deng $X$, Wang J, Hao X, Lau YC, Wong JY, Guan Y, Tan X, Mo X, Chen Y, Liao B, Chen W, Hu F, Zhang Q, Zhong M, Wu Y, Zhao L, Zhang F, Cowling BJ, Li F, Leung GM. Temporal dynamics in viral shedding and transmissibility of COVID-19. Nat Med 2020;26(5):672-5. DOI PubMed

10. Bai Y, Yao L, Wei T, Tian F, Jin DY, Chen L, Wang M. Presumed Asymptomatic Carrier Transmission of COVID-19. JAMA 2020;323(14):1406-7. DOI PubMed

11. Di Francesco RC. Mouth breathing: the ent doctor's point of view. J Bras Ortodon Ortop Facial. 1999;4(21):241-7. https://pesquisa.bvsalud.org/portal/resource/pt/lil-351457

12. Leboulanger $\mathrm{N}$. Nez bouché et bouche ouverte : le point de vue de l'ORL. Orthod Fr 2013;84(2):185-90.

DOI PubMed
13. Abreu RR, Rocha RL, Lamounier JA, Guerra AF. Etiology, clinical manifestations and concurrent findings in mouthbreathing children. J Pediatr (Rio J) 2008;84(6):529-35. DOI PubMed

14. Motta LJ, Alfaya TA, Marangoni AF, Ferrari RA, Fernandes KP, Bussadori SK. Gender as risk factor for mouth breathing and other harmful oral habits in preschoolers. Braz J Oral Sci 2015;11(3):377-80. https://periodicos.sbu. unicamp.br/ojs/index.php/bjos/article/view/8641374

15. Martel J, Ko YF, Young JD, Ojcius DM. Could nasal nitric oxide help to mitigate the severity of COVID-19? Microbes Infect 2020;22(4-5):168-71. DOI PubMed

16. Franková $\mathrm{V}$, Rychterová $\mathrm{V}$. Inhalatory infection of mice with influenza Ao/PR8 virus. II. Detection of the virus in the blood and extrapulmonary organs. Acta Virol 1975;19(1):35-40. PubMed 